\title{
An intriguing approach to the fractional Mellin transform method
}

\author{
Ozlem Ozturk Mizrak, Nuri Ozalp
}

Karabuk University, Department of Mathematics, Baliklarkayasi Mevkii 78050, Karabuk, Turkey

oomizrak@ankara.edu.tr

Ankara University, Department of Mathematics, 06100 Besevler, Ankara, Turkey

nozalp@science.ankara.edu.tr

\section{ABSTRACT}

We propose an adapted Mellin transform method that gives the solution of a fractional differential equation with variable coefficients in ordinary domain. After we mention a transformation of cosmic time to individual time (CTIT), we explain how it can reduce the problem from fractional form to ordinary form when it is used with Mellin transformation, via an example for $0<\alpha<1$; where $\alpha$ is the order of fractional derivative. Then, we give an application of the results.

Keywords: Mellin transform, fractional differential equation, the CTIT transformation, fractional derivative.

\section{Mathematics Subject Classification: 26A33, 34K17, 35A22}

\section{INTRODUCTION}

Mellin Transform has many application areas including digital data structures, probabilistic algorithms, asymptotics of Gamma-related functions, coefficients of Dirichlet series, asymptotic estimation of integral forms, asymptotic of algorithms and communication theory [1]. On the other side, in recent decades, non integer (fractional) differentiation has become a more and more popular tool for modeling physical systems from diverse areas such as heat flow [2], electrical circuits [3] [5], control [6]-[8] and medicine [9]. Thus the hug of Mellin analysis and fractional analysis was inevitable and in the literature, one can find so many fractional calculus applications that use Mellin transform as a solution method [10]-[13]. While some of them introduce an a-th order Mellin transform operator (M) in different forms, some use conventional Mellin transform operator (M) directly or but as far as we know, in the current literature, there is not any approach that gives Mellin transform of the $\alpha$-th derivative of a function in terms of Mellin transform of another function's integer order derivative. Such an approach means reducing the process of working with Mellin transform from fractional domain to integer domain of which theory is constructed with various advanced techniques when compared with the fractional theory. The main objective of this paper is to test if such an alternative treatment is possible rather than implementing Mellin transform method directly while finding the solution of a fractional dierential equation with variable coefficients.

In this context, the article is organized as follows: In Section 2, some preliminaries about fractional calculus and Mellin transform are presented. In Section 3, for the convenience of the reader, we mention about the motivation under the proposed Mellin transform method. Some main results are drawn in Section 4. Section 5 is devoted to an example to show the applicability of the adapted Mellin transform method. Finally, some conclusions are given in Section 6.

\section{PRELIMINARIES}

Definition 1. The Caputo type fractional derivative of order $\alpha>0$ of a func- tion $f:(0,1) \rightarrow R$ is defined by

$$
D^{\alpha} x(t)=\frac{1}{\Gamma(n-\alpha)} \int_{0}^{t}(t-\tau)^{n-\alpha-1} x^{(n)}(\tau) d \tau
$$

where $n-1$ is the integer part of $\alpha$.

Definition 2. Let $f(x)$ be locally Lebesgue integrable over $(0,1)$. The Mellin transform of $f(x)$ is defned by

$$
M[x(t) ; s]=f(s)=\int_{0}^{\infty} t^{s-1} x(t) d t .
$$

The largest open strip $\langle a, b\rangle$ in which the integral converges is called the fundamental strip. The inverse Mellin transform is defined as the following:

Definition 3. Let $f(x)$ be integrable with fundamental strip $\langle\alpha, \beta\rangle$. If $c$ is such that $\alpha<c<\beta$ and $f(s=c+i t)=M[f(x) ; s]$ is integrable, then the equality

$$
x(t)=\frac{1}{2 \pi i} \int_{c-i \infty}^{c+i \infty} M[x(t) ; s] t^{-s} d s
$$

holds almost everywhere. Moreover if $f(x)$ is continuous, then equality holds everywhere on $(0,+1)$. 
Definition 4. The Mellin convolution operator of two functions $f(t)$ and $g(t)$, given on $\mathbb{R}_{+}$, is defined for $x \in \mathbb{R}_{+}$ by the integral

that yields the form

$$
\text { (2.4) } x(t) * \phi(t)=\int_{0}^{\infty} x\left(\stackrel{t}{\tau}_{\tau}^{t}\right) \phi(\tau) \frac{d \tau}{\tau}
$$

$$
(2.5) \quad M(x * \phi)(s)=M(x)(s) M(\phi)(s) .
$$

\section{FROM TWO KINDS OF TIME TO A TRANSFORMATION METHOD}

It is generally known that as conventional calculus includes just integer order differential and integral operators, it significantly simplify its use for solving applied problems in various fields of science. However, in case of fractional calculus, it is not so, even if it represents a rapidly growing field both in theory and in applications to real world problems. Because of this, if a link between the ordinary domain and the fractional domain was provided, it would be ideal to facilitate the fractional order applications. Such a relation might be constructed on using two kinds of time: the individual time, $\tau$, (which is considered as flowing equably) and the cosmic time, $T$, (which flows non-equably). Transformation from Cosmic Time to Individual Time (CTIT) can be described by the equation

$$
\text { (3.1) } T=g_{t}(\tau)=\frac{1}{\Gamma(\alpha+1)}\left(t^{\alpha}-(t-\tau)^{\alpha}\right)
$$

or equivalently

$$
\text { (3.2) } \tau=t-\left(t^{\alpha}-T \Gamma(\alpha+1)\right)^{1 / \alpha} .
$$

This relationship between two time scales is used for giving a meaningful geometric and physical interpretation of fractional integration and fractional differentiation by the paper of Igor Podlubny and interested readers can find a detailed

information on these two kinds of time in [14]. By using above equality, a transformation method that gives the exact solution of a fractional differential equation in terms of the solution of the corresponding integer order differential equation is presented in [15]. $q$ extension of this method is also presented in [16]. Also, we have recently found a fractional Laplace transform method in the framework of this transformation [20]. Following the same methodology we generate a new adapted Mellin transform method which can be used to solve fractional dierential equations with variable coefficients in the form:

$$
\text { (3.3) } \sum_{k=0}^{m} A_{k} t^{\alpha+k}\left(D^{\alpha+k} x(t)\right)=f(t)
$$

where $D^{\alpha}$ represents the Caputo type fractional dierentiation, $\alpha>0, A_{k} \in \mathbb{R}$.

\section{MAIN RESULTS}

\section{Theorem 1.}

Let $f$ be Mellin transormable function defnned on $\mathbb{R}_{+}$. If differentiation under the integral sign is allowed, then we have

(1) $M\left[x^{(n)}(t) ; s\right]=\int_{0}^{\infty} x^{n}(t) t^{s-1} d t=\frac{(-1)^{n} \Gamma(s)}{\Gamma(s-n)} M[x(t) ; s-n][1]$.

(2) $M\left[t^{\beta} x(t) ; s\right]=M[x(t) ; s+\beta]$ where $\beta>0, \beta \in \mathbb{R}[17]$.

Mellin transform of a functions $\alpha$-th Caputo derivative is given by the following theorem in [18].

Theorem 2. Let $f$ be Mellin transformable defined on $\mathbb{R}_{+}$, and $f$ is a fractional derivative function for all $n-1<\alpha<n, n \in \mathbb{N}$, then:

(4.1) $M\left[x^{(\alpha)}(t) ; s\right]=\frac{\Gamma(s)}{\Gamma(s-\alpha)} M[x(t) ; s-\alpha]$. 


\section{Theorem 3.}

(4.2) $M\left[x^{(\alpha)}(t) ; s\right]=\frac{1}{\Gamma(n-\alpha)} M\left[\left(\frac{1}{t^{\alpha}-\Gamma(\alpha+1)}\right)^{1-\frac{(n-1)}{\alpha}} ; s\right] M\left[x_{*}^{(n)}\left(\frac{t^{\alpha}}{\Gamma(\alpha+1)}\right)\left(\frac{t^{\alpha}}{\Gamma(\alpha+1)}\right)^{\frac{(n-1)}{\alpha}} ; s\right]$ where $\alpha>0, n-1<\alpha<n, n=[\alpha]+1$ and $x_{*}(T)=x\left(t-\left(t^{\alpha}-T \Gamma(\alpha+1)\right)^{1 / \alpha}\right)$.

Proof. Let us use the induction method for the proof: First we should check the validity of the theorem for $n=1$. When one substitutes the equality (3.2) into the denition of $M\left(x^{(\alpha)}(t)\right)$, the equality (4.2) is easily obtained:

$$
\begin{gathered}
M\left(x^{(\alpha)}(t)\right)=\int_{0}^{\infty} t^{s-1}\left(\frac{1}{\Gamma(1-\alpha)} \int_{0}^{t}(t-\tau)^{-\alpha} x^{\prime}(\tau) d \tau\right) d t \\
=\frac{1}{\Gamma(1-\alpha) \Gamma(\alpha+1)} \int_{0}^{\infty} t^{s-1} \int_{0}^{t^{\alpha} / \Gamma(\alpha+1)} x_{*}^{\prime}(T)\left(\frac{t^{\alpha}}{\Gamma(\alpha+1)}-T\right)^{-1} d T d t \\
=\frac{1}{\Gamma(1-\alpha) \Gamma(\alpha+1)} \int_{0}^{\infty} t^{s-1}\left\{\left(\frac{\Gamma(\alpha+1)}{t^{\alpha}-\Gamma(\alpha+1)}\right) * x_{*}^{\prime}\left(\frac{t^{\alpha}}{\Gamma(\alpha+1)}\right) d t\right. \\
=\frac{1}{\Gamma(1-\alpha) \Gamma(\alpha+1)} M\left[\frac{\Gamma(\alpha+1)}{t^{\alpha}-\Gamma(\alpha+1)} ; s\right] M\left[x_{*}^{\prime}\left(\frac{t^{\alpha}}{\Gamma(\alpha+1)}\right) ; s\right] \\
=\frac{1}{\Gamma(1-\alpha)} M\left[\frac{1}{t^{\alpha}-\Gamma(\alpha+1)} ; s\right] M\left[x_{*}^{\prime}\left(\frac{t^{\alpha}}{\Gamma(\alpha+1)}\right) ; s\right]
\end{gathered}
$$

Now assume that the equality (4.2) is provided for $n=k$ where $k=2,3,4, \ldots$ Then is the equality (4.2) verificated for $n=k+1$ where $k=2,3,4, \ldots$ or is the equality

$$
\left.M\left[x^{(\alpha)}(t) ; s\right]=\frac{1}{\Gamma(k+1-\alpha)} M\left(\frac{1}{t^{\alpha}-\Gamma(\alpha+1)}\right)^{1-\frac{k}{\alpha}} ; s\right] M\left[x_{*}^{(k+1)}\left(\frac{t^{\alpha}}{\Gamma(\alpha+1)}\right)\left(\frac{t^{\alpha}}{\Gamma(\alpha+1)}\right)^{\frac{k}{\alpha}} ; s\right]
$$

true for $k<\alpha<k+1$ ?

As $D^{\alpha+m}(x(t))=D^{\alpha}(D x(t))$ where $m=0,1,2, \ldots$ and $n-1<\alpha<n$ (see (2.143) in [19]), $M\left[x^{(\alpha)}(t) ; s\right]$ can be written as $\left.M\left[\left(x^{(k)}\right)^{(\alpha-k)}(t)\right) ; s\right]$ where $k=2,3,4 \ldots$ and $k<\alpha<k+1$. Let say $x^{(k)}(t)=r(t)$. Then

$$
\begin{aligned}
& \left.M\left[x^{(\alpha)}(t) ; s\right]=M\left[\left(x^{(k)}\right)^{(\alpha-k)}(t)\right) ; s\right] \\
& =\int_{0}^{\infty} t^{s-1}\left(\frac{1}{\Gamma(k+1-\alpha)} \int_{0}^{t}(t-\tau)^{k-\alpha} r^{\prime}(\tau) d \tau\right) d t \\
& =\frac{1}{\Gamma(k+1-\alpha)(\Gamma(\alpha+1))^{1-\frac{k}{\alpha}}} \int_{0}^{\infty} t^{s-1} \int_{0}^{t^{\alpha} / \Gamma(\alpha+1)} r_{*}{ }^{\prime}(T)\left(\frac{t^{\alpha}}{\Gamma(\alpha+1)}-T\right)^{\frac{k}{\alpha}-1} d T d t \\
& =\frac{1}{\Gamma(k+1-\alpha)(\Gamma(\alpha+1))^{1-\frac{k}{\alpha}}} \int_{0}^{\infty} t^{s-1}\left\{\left(\frac{\Gamma(\alpha+1)}{t^{\alpha}-\Gamma(\alpha+1)}\right)^{1-\frac{k}{\alpha}} *\left(r_{*}\left(\frac{t^{\alpha}}{\Gamma(\alpha+1)}\right)\left(\frac{t^{\alpha}}{\Gamma(\alpha+1)}\right)^{\frac{k}{\alpha}}\right) d t\right. \\
& \left.=\frac{1}{\Gamma(k+1-\alpha)(\Gamma(\alpha+1))^{1-\frac{k}{\alpha}}} M\left[\frac{\Gamma(\alpha+1)}{t^{\alpha}-\Gamma(\alpha+1)}\right)^{1-\frac{k}{\alpha}} ; s\right] M\left[r_{*}{ }^{\prime}\left(\frac{t^{\alpha}}{\Gamma(\alpha+1)}\right)\left(\frac{t^{\alpha}}{\Gamma(\alpha+1)}\right)^{\frac{k}{\alpha}} ; s\right] \\
& =\frac{1}{\Gamma(k+1-\alpha)} M\left[\left(\frac{1}{t^{\alpha}-\Gamma(\alpha+1)}\right)^{1-\frac{k}{\alpha}} ; s\right] M\left[r_{*}{ }^{\prime}\left(\frac{t^{\alpha}}{\Gamma(\alpha+1)}\right)\left(\frac{t^{\alpha}}{\Gamma(\alpha+1)}\right)^{\frac{k}{\alpha}} ; s\right]
\end{aligned}
$$




$$
=\frac{1}{\Gamma(k+1-\alpha)} M\left[\left(\frac{1}{t^{\alpha}-\Gamma(\alpha+1)}\right)^{1-\frac{k}{\alpha}} ; s\right] M\left[x_{*}^{(k+1)}\left(\frac{t^{\alpha}}{\Gamma(\alpha+1)}\right)\left(\frac{t^{\alpha}}{\Gamma(\alpha+1)}\right)^{\frac{k}{\alpha}} ; s\right]
$$

and the proof is completed.

\section{EXAMPLE}

Example 1. To show the applicability of the proposed method we choose the simplest form of the equation (3.3) as

$$
\text { (5.1) } t^{\alpha} D^{\alpha} x(t)=\delta(t-a)
$$

where $0<\alpha<1, a \in \mathbb{R}$. Applying Mellin transform (4.2) to (5.1) and taking the properties (1) and (2) in Theorem 1 into account, we find the solution of the given fractional dierential equation as

$$
\text { (5.2) } x(t)=\frac{1}{2 \pi i} \int_{c-i \infty}^{c+i \infty} X_{*}(s) x^{-s} d s
$$

where $X_{*}(s)=\frac{a^{s-\alpha} \Gamma(1-\alpha) \alpha^{2} \Gamma(\alpha)}{(s-\alpha) \pi \cos e c\left(\frac{\pi(s+1)}{\alpha}\right)\left(-\frac{1}{\Gamma(\alpha+1)}\right)^{\frac{s+1}{\alpha}}}$.

\section{CONCLUSION}

In this paper, we propose an alternative way including conventional Mellin transform to solve the fractional differential equations with variable coefficients. Our proposed methodology produces the solution just by applying a transformation, namely the CTIT transformation, to the definition of a functions $\alpha$-th order derivative and reduces the problem into ordinary domain. Also, an illustrative example is given to provide the usage of the method.

\section{REFERENCES}

1. Kılıçman, A, A note on Mellin transform and distributions, Mathematical \& Computational Applications, VoL9, No. 1, pp. 65-72, 2004.

2. Y. Zhang, H. M. Srivastava and M. C. Baleanu, Local fractional variational iteration algorithm II for nonhomogeneous model associated with the non-differentiable heat flow, Advances in Mechanical Engineering, 2015, Vol. 7(10) 1-5.

3. A. Alsaedi, J. J. Nieto and V. Venktesh, Fractional electrical circuits, Advances in Mechanical Engineering, 2015, Vol. 7(12) 1-7.

4. A. Atangana, J. J. Nieto, Numerical solution for the model of RLC circuit via the fractional derivative without singular kernel, Advances in Mechanical Engineering, 2015, Vol. 7(10) 1-7.

5. A. Atangana, B. S. Alkahtani, Extension of the resistance, inductance, capacitance electrical circuit to fractional derivative without singular kernel, Advances in Mechanical Engineering, 2015, Vol. 7(6) 1-6.

6. Q. Gao, Y. Hou, T. Deng, C. Wang and R. Hou, Extended state observer based fractional order proportional-integral-derivative controller for a novel electro-hydraulic servo system with iso-actuation balancing and positioning, Advances in Mechanical Engineering, 2015, Vol. 7(12) 1-11.

7. Q. Gao, K. Li, Y. Hou, R. Hou and C. Wang, Balancing and positioning for a gun control system based on fuzzy fractional order proportional-integral-derivative strategy, Advances in Mechanical Engineering, 2016, Vol. 8(3) 1-9.

8. A. M. Lopes, J. T. Machado, Visualizing control systems performance: A fractional perspective, Advances in Mechanical Engineering, 2015, Vol. 7(12) 1-8.

9. Y. Zhang, S. Wang, G. Liu and J.Yang, Computer-aided diagnosis of abnormal breasts in mammogram images by weighted-type fractional Fourier transform, Advances in Mechanical Engineering, 2016, Vol. 8(2) 1-11. 


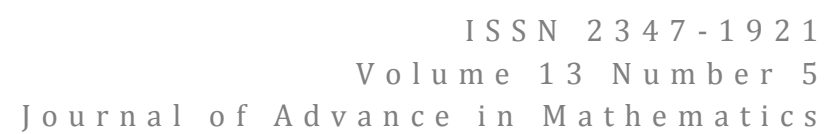

10. F. AlAzemi, A. AlAzemi, L. Boyadjiev, Mellin transform method for solving the Black- Scholes Equation, International Journal of Pure and Applied Mathematics, Volume 97 No. 32014, 287-301.

11. Magorzata Klimek, Daniel Dziembowski, On Mellin transform application to solution of fractional dierential equations, Scientic Research of the Institute of Mathematics and Computer Science, 2008, Volume 7, Issue 2 , pages 31-42.

12. O. Gonz'alez-Gaxiola, Jos'e A. Santiago, An $\alpha$-Mellin Transform and Some of its Applica- tions, Int. J. Contemp. Math. Sciences, Vol. 7, 2012, no. 48, 2353-2361.

13. Yanka Nikolova, $\alpha$-Mellin Transform and One of Its Applications, Mathematica Balkanica New Series Vol. 26, 2012, Fasc. 1-2.

14. I. Podlubny, Geometric and physical interpretation of fractional integration and fractional differentiation, Fractional Calculus and Applied Analysis 5 (4) (2002) 367-386.

15. E. Demirci, N. Özalp, A method for solving differential equations of fractional order, J. Comput. Appl. Math. 236, 2754-2762 (2012).

16. I. Koca, A method for solving dierential equations of $q$-fractional order, Applied Mathematics and Computation 266 (2015) 1-5.

17. Katugampola, U. N., Mellin Transforms of Generalized Fractional Integrals and Derivatives, Applied Mathematics and Computation 00 (2014) 1-17.

18. Adem Kılıçman, Maryam Omran, Note on fractional Mellin transform and applications, SpringerPlus (2016) 5:100 DOI 10.1186/s40064-016-1711-x.

19. I. Podlubny, Fractional Differential Equations, Academic Press, New York, 1999.

20. Nuri Ozalp, Ozlem Ozturk Mizrak, Fractional Laplace transform method in the framework of the CTIT transformation, Journal of Computational and Applied Mathematics (2016), DOI: 10. 1016/j.cam. 2016.11.039.

\section{BIBLIOGRAPHY}

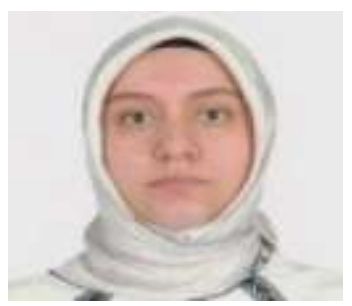

Ozlem Ozturk Mizrak received the B.Sc. and M.Sc. degrees in 2010 and 2013 from the Department of Mathematics from Gazi University, Turkey respectively. She is still doing doctorate at Ankara University with the thesis title "On Some Fractional Dynamic Models". She is also working as a research assistant at Department of Mathematics of Karabuk University. Her research interests are fractional differential equations, mathematical modelling and mathematical oncology.

This work is licensed under a Creative Commons Attribution 4.0 International License. 